\title{
Using Prescriptive Analytics to support the Continuous Improvement Process
}

\author{
Günther Schuh ${ }^{1}$, Jan-Philipp Prote ${ }^{1}$, Thomas Busam ${ }^{20000-0003-0221-4443], ~}$ \\ Rafael Lorenz ${ }^{3[0000-0001-7473-3328]}$ and Torbjörn H. Netland ${ }^{3[0000-0001-7382-1051]}$ \\ ${ }^{1}$ Laboratory for Machine Tools and Production Engineering (WZL), \\ RWTH Aachen University, 52074 Aachen, Germany \\ ${ }^{2}$ Schuh \& Co. GmbH, Campus Boulevard 57, 52074 Aachen, Germany \\ thomas.busameschuh-group.com \\ ${ }^{3}$ Department of Management, Technology, and Economics, \\ ETH Zurich, 8092 Zurich, Switzerland
}

\begin{abstract}
The continuous improvement process (CIP) enables companies to increase productivity constantly by sourcing ideas from their employees on the shop floor. However, shorter production cycles require manufacturing companies to also adapt their production processes in a faster manner and reduce resources for CIP activities. Traditional CIP approaches fall short in such a fastpaced environment characterized by uncertainty. This study proposes a novel approach for increasing the efficiency and speed of the CIP by using data of previous improvements and predict current potentials. This results in a prescriptive model supporting the employees how to improve their processes.
\end{abstract}

Keywords: Continuous Improvement Process; Data-driven Production Management; Knowledge Management

\section{Introduction}

Manufacturing companies are required to constantly improve their capabilities to increase their productivity in the future. A main driver for increasing the productivity on a day-to-day basis is the continuous improvement process (CIP), which is at the heart of any manufacturing organization [1]. In the popular illustration of the Toyota Production System (TPS), for instance, the CIP stands in the center of the illustrated house and receives special focus [2]. The CIP helps to access and use the knowledge and suggestions of the employees to constantly question the current processes.

However, not only the required capabilities itself change - also the rate at which this change is happening increased within the last years. Customers are asking for more individualized products for the same costs as mass-produced products. Digital technologies allow for needed productivity gains outside current limits. The usage of these technologies will significantly change the way manufacturing companies produce products. Such significant changes of the process, so called process innovations, will induce highly cyclical changes until new stable production conditions are achieved. 
In order to ensure increasing productivity under such conditions, the CIP needs to be enhanced to overcome its time consuming latencies. Therefore, digitalization offers new opportunities for collecting and analyzing huge amount of data in a short time that a human could not handle. Hence, this paper proposes a model to enhance the CIP with digital capabilities to leverage its potential for future productivity gains.

\section{Definition and Challenges of the Continuous Improvement Process}

The CIP is rooted in the literature on lean management and total quality management (TQM) [3]. It is a method of constantly developing processes further towards an overarching goal [4]. This goal serves as a direction and guides the improvement actions. The employees perform these actions continuously along the way.

The CIP is often taken as a synonym for Japanese term kaizen. This term is first introduced by Imai [5] who further differentiates between three levels of kaizen: management, group, and individual. The focus of kaizen is the incremental, iterative, and continuous improvements of a system [6]. In contrast, the literature defines the term kaikaku as step-wise improvements [7]. These happen for instance with the introduction of a new manufacturing technology.

Whereas in earlier years companies tried to operationalize the CIP through tools and rigor meetings, companies recently acknowledge the importance of the softer aspects; For achieving sustainable results the CIP needs to be anchored in the company's culture [1]. Therefore, the key resource of the CIP are the employees [2]. Not only do they shape and influence the culture, but they also possess the specific knowledge about the process and are the problem solvers. This is critically as the CIP is not a top-down approach but lives throughout the organization.

With an increasing complexity of the processes due to quicker cycles of different products, traditional continuous improvement falls short on quickly identifying the root causes. Digitalization allows for the collection and analysis of a huge amount of data to manage such complex processes. However, incorporating information technology into the manufacturing process can detach the employee from the problem solving process: they might lack the expertise for analyzing the data needed to understand the process. Thus, data that can help in improving the processes remains far too often unexplored.

The performance and financial benefit of the improvement activities are not always clear. In a case study of two plants for instance the annual costs for the continuous improvement investment exceeded the annual cost savings from it for one plant [8]. The financial pressure increases when island solutions are developed. If a similar problem has already been solved in another line or another plant, the company could take advantage of scaling the solution throughout the organization. However, if the knowledge about the presence of these improvements is not shared, the company will develop redundant improvements that create a non-standardized infrastructure [9].

Following the literature, the ability to minimize the latency times regarding acquiring and analyzing data, as well as decision making towards the final implementation 
is key to efficient continuous improvement in a frequently changing production environment. To reach that goal, an approach is required that meets the requirements for the future continuous improvement developed above, namely:

- Low individual effort during the process chain of analyzing data until decision making in a fast pace to preempt frequent changes of the production environment.

- Real improvement of processes instead of simple failure prediction and handling.

- Making use of the organization-wide knowledge.

\section{State of the Art}

Most of the different approaches for continuous improvement follow two different streams, namely management- or data-driven. The management- (or experiencedriven) CIP is the most researched one. The data-driven approach is growing fast due to new opportunities that come along with Industry 4.0. In the following, existing continuous improvement approaches for both streams are reviewed and evaluated.

\subsection{Discussion of Existing Approach}

Regarding management and experience driven CIP, Rother [4] describes the "Toyota Kata" as the currently most prominent approach. Newer explanations of this approach like "Toyota Kata Culture" or "Leading improvements successfully" share the same concept, that, based on a vision, the current state has to be improved towards a target condition. Working one's way up on that path will reveal many obstacles, which have to be addressed step by step in the problem solving cycle (respectively the PDCAcycle). This approach is executed by the employees, driven to learn how to improve their processes daily. In an addition to that, those problem solvers embody the first link of a hierarchy of coachees and coaches, way up to the plant manager. Rother [4] recommends this "Coaching Kata" to further enhance the problem solving ability of the coachees as well as providing a quick escalation and plant overarching goal setting. Hence, those two aspects make this approach very time and resource intensive when applied thoroughly $[4,10,11]$.

In the field of data driven improvement, Groeger et al. [12] develop prescriptive analytics for recommendation-based business process optimization at process runtime. Using data mining, the quality output of a product can be predicted via a decision tree for specific processes. The discovered rules of the decision tree are then translated into recommended actions. If during an executed process a metric deviation is predicted, these actions should be taken. This approach focuses on quality issues and how to avoid them, but does not address the challenge of how to improve a process regarding time nor cost. Kassner and Mitschang incorporate unstructured information like pictures or text descriptions into a decision support to handle exceptions on the shop floor. Their described architecture "MaXCept" does this in 5 steps: exception recognition, exception classification, exception escalation, solution recommendation and task-expert matching [13]. Although being intended to be automated and in real- 
time, their approach is still depending on experts and focuses on solving operational problems on the shop floor rather than addressing process improvement. Ringsquandl et al. [14] introduce a graph-based analytics framework derived from a comprehensive requirement analysis in order to reduce the customization efforts which come along with most advanced manufacturing analytics. Being developed on an architectural level, this approach still requires adjustments to consider extensive domain knowledge like constraints or relations of a specific process into the algorithm [14]. Wuest [15] provides a method applying Supervised Machine Learning to the "product state concept" by incorporating product and process inter- and intra-relations rather than only focusing on individual processes. Using product states consisting of a set of state characteristics enables a product oriented view of the physical value stream. Those characteristics can be altered by process inter- and intra-relations, the so called state drivers. Wuest incorporated this element into the analysis through applying support vector machine-based feature ranking. The results of this method can be used for e.g. quality monitoring and advanced process control. However, this concept is still depending on expert knowledge when it comes to incorporating implicit process interand inter-relations.

\subsection{Deficits of Existing Approaches and Summary}

Management and experience driven approaches on the one hand take up too much resources of employees and time regarding the described challenges in the previous chapter. Data driven approaches on the other hand lag behind when the production environment is changing too quickly. The effort to design and implement a new improvement system or application exclusively for the changed process is surpassing the benefits of the achievable improvements, given the limited time until the same process changes once more [15]. Moreover, the existing approaches focus on predicting quality instead of recommending real process improvement. To overcome the challenges of both streams, a new approach for an efficient continuous improvement process in an unstable and therefore frequently changing production environment is proposed in the following.

\section{Approach for a data-driven continuous improvement process}

The novel approach presented in this chapter combines the advantages of the previously described two directions to meet the requirements stated in the theoretical background. Fig. 1 illustrates the three phases of the approach. The aim is to provide tailored solution suggestions to a responsible employee who has chosen a specific process to focus on. Artificial intelligence is used to choose suggestions from the database of previous suggestions. The required input besides already conducted improvements are the organization-wide targets, respectively the broken down ones for the chosen process and its parameters. The following describes the approach of each of the three phases. 


\begin{tabular}{|c|c|c|}
\hline I: Description phase & II: Modelling phase & III: Suggesting phase \\
\hline $\begin{array}{l}\text { Aim: } \\
\text { - Structured and } \\
\text { implemented data base }\end{array}$ & $\begin{array}{l}\text { Aim: } \\
\text { - Trained predicting model }\end{array}$ & $\begin{array}{l}\text { Aim: } \\
\text { - Derivation and suggestion } \\
\text { of improvement activities }\end{array}$ \\
\hline $\begin{array}{l}\text { Procedure: } \\
\text { - Evaluation of digital } \\
\text { maturity } \\
\text { - Determination and } \\
\text { preparation of data }\end{array}$ & $\begin{array}{l}\text { Procedure: } \\
\text { - Retrospective } \\
\text { determination of cause- } \\
\text { effect relationships } \\
\text { - Training and testing }\end{array}$ & $\begin{array}{l}\text { Procedure: } \\
\text { - Implement pattern } \\
\text { recognition of user stories } \\
\text { - Integrate data base and } \\
\text { predicting model }\end{array}$ \\
\hline
\end{tabular}

Fig. 1. Three phases for identifying suggested improvement action

\subsection{Description Model for the Determination and Preparation of Data}

The aim of this first phase is to determine the required data and format to prepare this information as an input for the subsequent phases. Therefore, the digital maturity is evaluated based on the index developed by Schuh et al. [16]. to detect the required changes on the quality and availability of the company's process data. Only when the company is ranked on level three or higher the procedure can deliver robust improvements. This level requires an already digitized production while collecting and visualizing data in a manufacturing execution system (MES) [16].

As illustrated in Fig. 2, this phase connects the available process data of the current state with situational data of the respective process environment (e.g., room temperature, humidity, etc.). This information has to be stored together as an event log to be accessible by the system for later comparison with other event logs or choosing the next process to be improved. A complete event log consists of a process use-case, describing the current condition with parameters, KPIs and the respective targets for a specific process, data of the environment, improvement idea and the corresponding results. These often separately stored information can be linked via timestamps and identification numbers. Whenever an improvement idea is performed, it will be saved as a new event log to be available in the constantly growing organization-wide database. Ideas of the past will also be imported likewise.

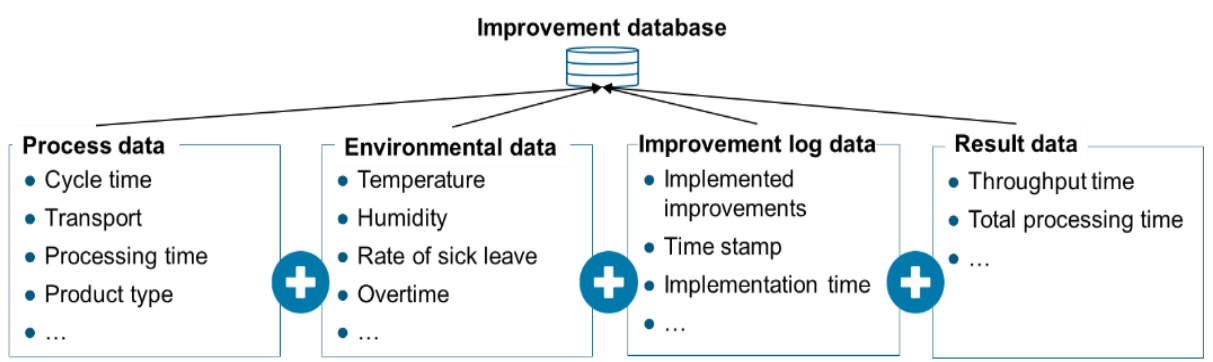

Fig. 2. Logic of the merged database of improvement data 


\subsection{Extended Effect Model for Retrospective Determination of Cause- Effect Relationships}

One of the objectives of the model in the second phase is to discover unknown or implicit cause-and-effect relations of the production processes. To find these relations and thereby foster better improvement ideas, the second phase integrates the knowledge discovery in databases (KDD) process as described by Fayyad et al. [17] to feed the model. Thereby, unknown relationships between different processes or parameters are added to a library of already known manufacturing-relevant causeand-effect relationships, for instance provided by Hopp and Spearman [18]. With these discovered relationships new improvement ideas can already be developed at this stage. The results of past improvements will also be added in the suggested format described in chapter 4.1 to the collection of formulas. The formulas in the library are incorporated into a multi-variant model to work as a digital shadow, which can also be used to forecast situations that have never existed before. Instead of simply feeding and training the multi-variant model with process data, this approach suggests to explicitly train it with the extracted formulas of the library and past improvements in an artificial generated data-set upfront. Later on, the preconditioned model will continue learning currently unknown relations on its own, especially by taking environmental data into account. This way a neural network for instance can focus on new relations in the real data-set instead of having to learn already known relations or formulas from the start.

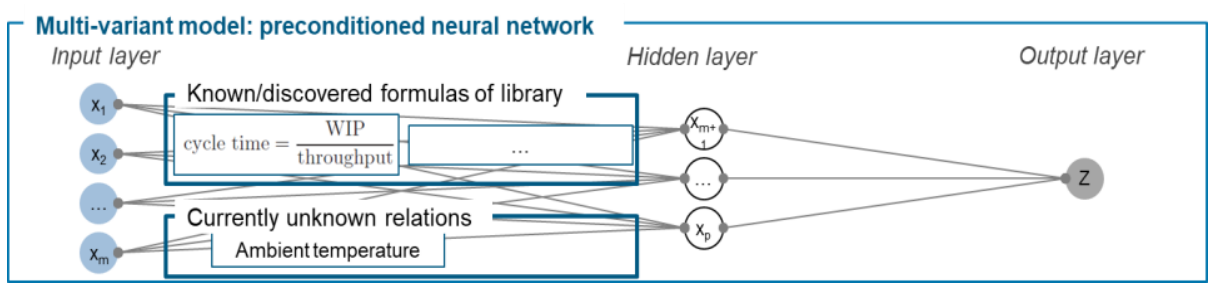

Fig. 3. Structure of the preconditioned multi-variant model

Figure 3 shows the basic structure of the multi-variant model. The previously introduced model provides process and environmental data as information on the input layer as well as result data on the output layer.

\subsection{Explanation Model for Pattern Recognition of User Stories and Derivation of Improvement Activities}

The model of the third phase serves as an indirect interface to the person in charge to improve a specific process. The aim is to suggest the most suitable improvement and its effects, which can be visualized via an individually designable app on a portable devise as the direct interface. The suggestion is based on a similarity analysis between the process to be improved and the event $\log$ s in the organization-wide database of already implemented improvements. This phase begins with the description of the actual problem of the production process. The KPIs and parameters of the current 
state in contrast to the targets reveal a delta and are consolidated in a set of numbers similar to an event $\log$ as shown in figure 4 on the right side of the input vectors.

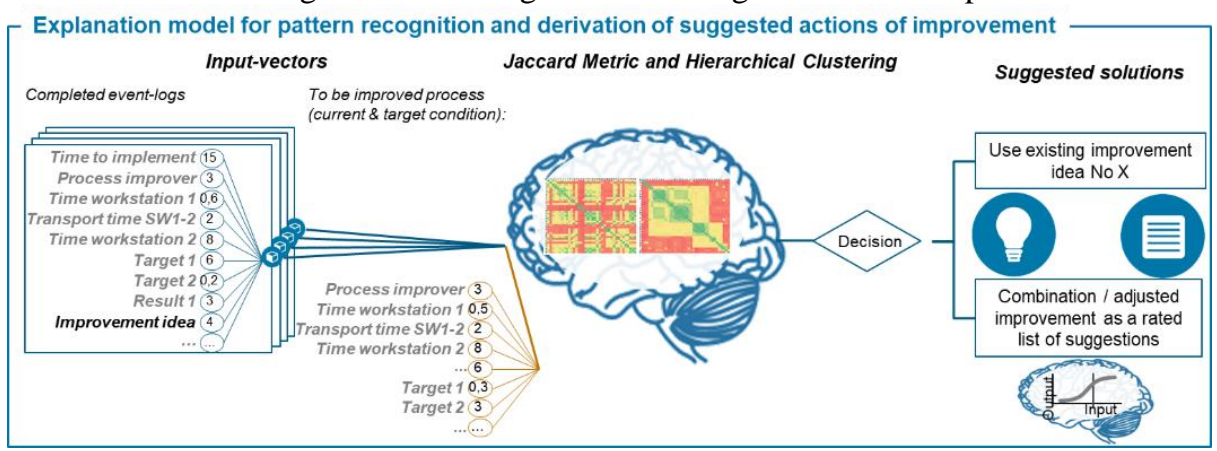

Fig. 4. Logic of the model to suggest improvement actions

This vector is then compared with the vectors of all event logs in the database. This comparison is performed by calculating the distance based on the Jaccard Metric, followed by applying hierarchical clustering to identify clusters of similarities. These will select the most similar event log by comparing the vector of the selected process and the ones of all event logs. Thereby, similar processes with the smallest vectorvariations (e.g., parameters, KPIs, targets or environment data) are suggested. The effect of those variations on the future result are then predicted by making use of the second model. The final model might also suggest applying two different improvement ideas at the same time if the delta between the current and the target state is too big for only one existing improvement solution. The hierarchical clustering allows the person in charge to choose from a set of different options suggested by the model. After implementing one of the suggested improvement ideas, the idea and its outcome are integrated into the database and constitute to a new event log.

\section{Conclusion}

Given the opportunities of Industry 4.0, the continuous improvement process can hugely benefit by incorporating the right algorithms and an advancement of the methodology. Besides improving required efforts this approach also enables an easy way to harvest historical data for recommendations and to turn implicit or even unknown process knowledge systematically into explicit organizational knowledge. If not yet documented, this approach helps to start filling up the organizations database with first improvement ideas in a structured format, which of course will take some time to reach a beneficial quantity. Further research should focus on the industrial application of this approach, which initially requires a detailed design of how to realize the three phases, and identify contingencies for different environments and industries. 


\section{References}

1. Anand, G, Ward, PT, Tatikonda, MV et al. (2009) Dynamic capabilities through continuous improvement infrastructure. Journal of Operations Management 27(6): 444-461

2. Liker, JK (2004) The Toyota way: 14 management principles from the world's greatest manufacturer. McGraw-Hill, New York, NY

3. Lam, M, O'Donnell, M, Robertson, D (2015) Achieving employee commitment for continuous improvement initiatives. International Journal of Operations \& Production Management 35(2): 201-215

4. Rother, M (2010) Toyota Kata: Managing people for improvement, adaptiveness, and superior results. McGraw-Hill, New York

5. Imai, M (1986) Kaizen: The key to Japan's competitive success. McGraw-Hill, New York

6. Brunet, AP, New, S (2003) Kaizen in Japan: an empirical study. International Journal of Operations \& Production Management 23(12): 1426-1446

7. Bertagnolli, F (2018) Lean Management: Einführung und Vertiefung in die japanische Managementphilosophie. Springer Gabler, Wiesbaden

8. Butler, M, Szwejczewski, M, Sweeney, M (2018) A model of continuous improvement programme management. Production Planning \& Control 29(5): 386-402

9. Lorenz, R, Lorentzen, K, Stricker, N et al. (2018) Applying User Stories for a customerdriven Industry 4.0 Transformation. IFAC-PapersOnLine 51(11): 1335-1340

10. Rother, M, Aulinger, G (2017) Toyota kata culture: Building organizational capability and mindset through kata coaching. McGraw-Hill, New York

11. Kamberg, M (2016) Die Verbesserungskata. Verbesserung erfolgreich führen, mit der Toyota-Kata und Lean Management Prozesse verbessern und Mitarbeiter entwickeln / von Marco Kamberg ; Band 1. CETPM Publishing, Herrieden

12. Gröger, C, Schwarz, H, Mitschang, B (2014) Prescriptive Analytics for Recommendation-Based Business Process Optimization. In: Abramowicz W (ed) Business information systems: 17th International Conference, BIS 2014, Larnaca, Cyprus, May 22-23, 2014 ; proceedings, vol 176. Springer, Cham, pp 25-37

13. Kassner, LB, Mitschang, B (2015 - 2015) MaXCept -- Decision Support in Exception Handling through Unstructured Data Integration in the Production Context: An Integral Part of the Smart Factory. In: 2015 48th Hawaii International Conference on System Sciences. IEEE, pp 1007-1016

14. Ringsquandl, M, Lamparter, S, Lepratti, R (2016 - 2016) Graph-based predictions and recommendations in flexible manufacturing systems. In: IECON 2016 - 42nd Annual Conference of the IEEE Industrial Electronics Society. IEEE, pp 6937-6942

15. Wuest, $T$ (2015) Identifying product and process state drivers in manufacturing systems using supervised machine learning. Springer theses. Springer, Cham

16. Schuh, G, Anderl, R, Gausemeier, J et al. (2017) Industrie 4.0 Maturity Index - Die digitale Transformation von Unternehmen gestalten. acatech

17. Fayyad, U, Piatetsky-Shapiro, G, Smyth, P (1996) From Data Mining to Knowledge Discovery in Databases. AI Magazine 17(3): 37-54

18. Hopp, WJ, Spearman, ML (2008) Factory physics, 3. ed., internat. ed. The McGrawHill/Irwin series. McGraw-Hill/Irwin, Boston 